\title{
O emprego dos contraceptivos orais combinados na síndrome dos ovários policísticos
}

\author{
The use of combined oral contraceptives in the polycystic ovary \\ syndrome
}

A síndrome dos ovários policísticos (SOP) é um distúrbio no eixo neuro-endócrinoreprodutor relacionado com alteração morfológica dos ovários e com produção androgênica elevada. Sua prevalência varia entre 6 e $12 \%$ da população feminina durante o período reprodutivo ${ }^{1-3}$. Deve-se salientar que a característica mais marcante nessa afecção é o hiperandrogenismo com anovulação crônica, sendo, por isso, designada de "anovulação crônica hiperandrogênica" por melhor descrever a síndrome ${ }^{1-3}$. Sua etiopatogenia é ainda muito debatida. Contudo, alguns investigadores apontam a ocorrência de distúrbio do metabolismo de carboidratos, principalmente da insulina. Esse fato é apoiado na alta prevalência de hiperinsulinemia nas mulheres com SOP, atingindo cifras de aproximadamente $70 \% \%^{4-6}$.

Salienta-se, ainda, que a obesidade piora a resistência insulínica em mulheres com SOP. Além disso, aumentaria o risco para cânceres da mama e do intestino grosso, diabetes melitus tipo 2, bem como predisporia ao incremento da frequência de doenças carviovasculares, como hipertensão arterial sistêmica, doença coronariana, acidente vascular encefálico ${ }^{7}$. Ademais, a anovulação pode determinar sangramento uterino anormal e lesões precursoras do câncer endometrial. Por essas razões, o adequado acompanhamento e aconselhamento das pacientes com SOP é fundamental para evitar as complicações e os eventuais riscos relacionados a essa síndrome. Assim, recomenda-se o emprego de progestagênio na segunda fase do ciclo ou contraceptivo hormonal combinado para amenizar a disfunção menstrual e o risco de desenvolver carcinoma do endométrio.

Os anticoncepcionais combinados são empregados em pacientes anovuladoras com hiperandrogenismo, pois os estrogênios diminuem os níveis androgênicos circulantes ao incrementar os níveis séricos de SHBG e diminuir a atividade da $5 \alpha$-redutase. Esta última é também determinada pelo progestagênio, o qual ainda inibe a síntese e a secreção de gonadotrofinas hipofisárias. Caracteriza o "repouso gonadal". Contudo, as mulheres com SOP têm alta prevalência de aterosclerose subclínica, refletindo na disregulação da função endotelial, bem como em anomalias na coagulação e no sistema fibrinolítico, aumentando o risco de fenômenos tromboembólicos ${ }^{8}$. Por essa razão, alguns autores acreditam que a escolha de anticoncepcionais combinados representa um desafio para o clínico?.

Sabe-se que os contraceptivos hormonais orais são amplamente empregados para as mulheres com SOP, mas há relatos de seu impacto sobre o metabolismo de carboidratos e

Disciplina de Endocrinologia Ginecológica do Departamento de Ginecologia da Universidade Federal de São Paulo - São Paulo (SP), Brasil.

Professor e Vice-Chefe da Disciplina de Endocrinologia Ginecológica do Departamento de Ginecologia da Universidade Federal de São Paulo - UNIFESP - São Paulo (SP), Brasil.

2 Professor Titular da Disciplina de Ginecologia do Departamento de Obstetrícia e Ginecologia da Faculdade de Medicina da Universidade de São Paulo - UNIFESP - São Paulo (SP), Brasil. 
lipídeos ${ }^{9}$. De fato, alguns autores sugerem que os anticoncepcionais combinados de baixa dose seriam os preferenciais, sobretudo, para as mulheres com resistência à insulina e dibetes mellitus não complicado. Contudo, esses autores recomendam o uso de progestagênio isolado quando há antecedentes de tromboembolismo ou a paciente tem hipertensão arterial sistêmica?

Deve-se ter cuidado especial com as mulheres com dislipidemia, pois os contraceptivos hormonais orais combinados podem elevar os níveis de triglicerídeos. Sabe-se que a hipertrigliceredemia constitui importante fator de risco para doença coronariana, em especial, em mulheres com distúrbio do metabolismo dos carboidratos, como as pacientes com diabete melito tipo $2^{10}$. Portanto, recomenda-se precaução no emprego de qualquer contraceptivo oral combinado.

Em relação ao risco de tromboembolismo venoso, há um grande estudo que avaliou mais de 10 milhões de mulheres dinamarquesas entre 15 e 49 anos de idade, sendo mais de três milhões usuárias de contraceptivos orais, no período de 1995 a $2005^{11}$. Investigou-se o risco de tromboembolismo em usuárias com diferentes tipos de contracepção hormonal, bem como o tipo de composição estroprogestativa, o tipo de progestagênio e a via de ministração dos contraceptivos. Verificou-se que aproximadamente $49 \%$ das mulheres com eventos de tromboembolismo venoso eram usuárias de contraceptivos hormonais orais, as quais tiveram o dobro do risco absoluto em comparação as mulheres não usuárias $(6,2$ contra 3,1 por 10.000 mulheres por ano), sendo o risco mais elevado no primeiro ano de uso do contraceptivo hormonal mesmo com a dose mais baixa de estrogênio. Em relação aos progestagênios, os norderivados de primeira e segunda geração (noretisterona, norgestimato e levonorgestrel) estiveram associados às menores porcentagens comparativamente com os de terceira geração (gestodeno, desogestrel), derivados da espirolactona (drosperinona) e da pregnana (ciproterona). Não foi registrado aumento do risco com o uso de contraceptivo com apenas progestagênio com levonorgestrel, noretisterona ou desogestrel, bem como o uso de dispositivo intrauterino com liberação hormonal ${ }^{11}$. Nesse estudo, não foram avaliadas, isoladamente, as mulheres com anovulação crônica e risco cardiovascular. Portanto, preconiza-se cuidados especiais ao se prescrever contraceptivos hormonais orais combinados em pacientes com SOP.

\section{Referências}

1. Carmina E, Lobo RA. Polycystic ovary syndrome (PCOS): arguably the most common endocrinopathy is associated with significant morbidity in women. J Clin Endocrinol Metab. 1999;84(6):1897-9.

2. Alvarez-Blasco F, Botella-Carretero Jl, San Millán JL, Escobar-Morreale HF. Prevalence and characteristics of the polycystic ovary syndrome in overweight and obese women. Arch Intern Med. 2006;166(19):2081-6.

3. Diamanti-Kandarakis E, Kouli CR, Bergiele AT, Filandra FA, Tsianateli TC, Spina GG, et al. A survey of the polycystic ovary syndrome in the Greek island of Lesbos: hormonal and metabolic profile. J Clin Endocrinol Metab. 1999;84(1 1):4006-11.

4. Khoury MY, Baracat EC, Pardini DP, Haidar MA, da Motta EL, de Lima GR. Polycystic ovary syndrome: clinical and laboratory evaluation. Sao Paulo Med J. 1996; $114(4): 1222-5$.

5. Motta EL, Baracat EC, Haidar MA, Juliano I, Lima GR. Ovarian activity before and after gonadal suppression by GnRH-a in patients with polycystic ovary syndrome, hyperandrogenism, hyperinsulinism and acanthosis nigricans. Rev Assoc Med Bras. 1998;44(2):94-8.

6. Maciel GA, Soares Júnior JM, Alves da Motta EL, Abi Haidar M, de Lima GR, Baracat EC. Nonobese women with polycystic ovary syndrome respond better than obese women to treatment with metformin. Fertil Steril. 2004;81 (2):355-60.

7. Baracat EC, Soares-Junior JM. Ovários policísticos, resistência insulínica e síndrome metabólica. Rev Bras Ginecol Obstet. 2007;29(3): 117-9.

8. Mak W, Dokras A. Polycystic ovarian syndrome and the risk of cardiovascular disease and thrombosis. Semin Thromb Hemost. 2009;35(7):613-20.

9. Soares GM, Vieira CS, de Paula Martins W, Dos Reis RM, de Sá MF, Ferriani RA. Metabolic and cardiovascular impact of oral contraceptives in polycystic ovary syndrome. Int J Clin Pract. 2009;63(1):160-9.

10. Ahmad J, Hameed B, Das G, Siddiqui MA, Ahmad I. Postprandial hypertriglyceridemia and carotid intima-media thickness in north Indian type 2 diabetic subjects. Diabetes Res Clin Pract. 2005;69(2):142-50.

11. Lidegaard $\varnothing$, Løkkegaard E, Svendsen AL, Agger C. Hormonal contraception and risk of venous thromboembolism: national follow-up study. BM. 2009;339:b2890. 